\title{
TRABALHOS PUBLICADOS
}

\section{PELO PROF. DR. PAULO SAWAYA}

1. Ensaios de Paleografia. Rev. Med., 10:61-72. São Paulo. Em col. F. M. Campos e O. M. Souza.

2. Duas anomalias musculares. Rev. Med., 8(39):37-48, 1926. São Paulo. Em col. O. M. Souza, J. T. Quino e J. F. Sainati.

3. Capilares sanguíneos intra-epiteliais da mucosa da uretra masculina humana. Bol. Soc. Med. Cir. São Paulo, 11(6-7):6, 1928. São Paulo. Em col. O. M. Souza.

4. Contribuição para o estudo da mucosa uretral humana. Tese de doutoramento, 70 pp., 8 tab. São Paulo.

5. Contribuições para o estudo da mucosa uretral humana. An. Fac. Med. S. Paulo, 5, 1930. São Paulo. (Em col. O. M. Souza).

6. Anotações craneológicas. An. Fac. Med. S. Paulo, 10(2):197-219, 1934. São Paulo.

7. Relatório de Botânica e Zoologia. Apresentado em março de 1934 ao Sr. Secretário da Educação e Saúde Pública.

8. Diretrizes do Ensino da Zoologia na Universidade de São Paulo. An. Fac. Fil. Ci. Letr., 1934-1935:73-89. São Paulo.

9. Prof. Ernst Bresslau (traços biográficos). An. Fac. Fil. Ci. Letr. Univ. S. Paulo, 1934-1935:245-254. São Paulo.

10. Ernst Bresslau. In memorium. Rev. Biol. Hig., 6(1):1-6, 1935. S. Paulo.

11. Alguns aspectos da biologia dos saguis. Bol. Biol. (n.s.), 2(4):141-149, 1936. São Paulo.

12. Sobre a placenta bidiscoidal de Hapale jacchus (1). Rev. Biol. Hig., y(1): 53-58, 1936. São Paulo.

13. Relaçōes da "Pars Thoraxica Tracheae" no Bradypys tridactylus L. Rev. Otorrinol., 4(5), t. 1-3, 1936. São Paulo.

14. A "Cobra cega" (Siphonops annulatus Mikan) como alimento da "Mussurana" (Pseudoboa doelia) (Daud.). Rev. Biol. Hig.,7:80-85, 1936. S. Paulo.

15. História Natural (Zoologia). Rev. Fac. Fil. Ci. Letr., n. ${ }^{\circ}$ 3:11-17, 1936. São Paulo.

16. Sobre o gênero Siphonops-Wagler (1928. Anphibio-Apoda com descrição de duas variedades novas. S. annulatus (Mikan) var. matmoratus e S. paulensis Boettg, var. maculatus. Bol. Fac. Fil. Ci. Letr. U.S.P., Zool., 1:225-263. t. 30-32, 1937. São Paulo.

Número especial em homenagem ao Prof. Dr. Paulo Sawaya, no ano jubilar de seu magistério. 
17 Ocorrência do Branchiostoma (Amphioxus) na baia de Santos. Bol. Biol. n.S., 3(2):43-46, 1937. S. Paulo (Em col. J. P. Carvalho).

18. Dis Blindwhile Siphonops (Mikan) als nahrung der "Mussuarama", Pseudoboa (Daud.) Zool. Anz., 118(5-6), 1937.

19. Introdução ao estudo da Fisiologia Comparativa. An. Fac. Fil. Ci. Letr., pp. 45-47, 1937-1938. São Paulo.

20. Inđicações sobre a orientação do Beija-flor (Argytrina sp.) e do mergulhão (Sula leucogaster Bodd), em relação ao ninho. Bol. Biol. n.s., 3(2):62-66, 1938. São Paulo.

21. Bemerkungen über die Orientierung des Kolibris (Argytrina sp.) und des Tölpels (Sula leucogaster Bodd) an Nest. Zool. Anz., 122(3-4):108-110, 1938.

22 Prof. Alfonso Bovero. Bol. Biol. n.s., 3(2):72-75, 1938. São Paulo

23. Sobre o "piolho da baleia" (Cyamus ovalis e C. erraticus Roussel de Vauzéme, 1934). Bol. Fac. Fil. Ci. Letr. USP. Zool., 2:197-268, 9 tab., 1938. São Paulo.

24. Proteção à fauna e à flora. Bol. Biol. n.s., 3(3):178-180, 1938. S. Paulo

25. Animais cavadores da praia arenosa. Arq. Inst. Biol., 10(supl.):319-326, 1939. São Paulo.

26. Sobre a mudança de cor nos Crustáceos. Bol. Fac. Fil. Ci. Letr. Zool., 3:1-109, t. 1-4, 1939. São Paulo.

27. Tradução do livro "Darwin" de Julian Huxley. 208 pp. Liv. Martins, 1940. São Paulo.

28. Sobre a histofisiologia dos órgãos excretores de alguns insetos. I. Orthoptera. Rev. Entomol., 1(1-2):231-252. Rio de Janeiro, 1940.

29. Sobre o poder venenoso das flechas dos índios Tucanos. Bol. Fac. Fil. Ci. Letr. Zool., 4:22-27, 1940. São Paulo.

30. Sobre o veneno das glândulas cutâneas, secreção e coração de Siphonops annulatus. Bol. Fac. Fil. Ci. Letr. Zool., 4:207-270, 1940. São Paulo.

31. Sobre a presença de um segundo seio venoso no coração do Anfíbio Siphonops annulatus (Mikan). Arq. Mus. Paranaense, 1(6):131-136, 1941. Curitiba.

32. Ritimos da vida animal. Alambique 2(5):1-2, 1941. São Paulo.

33. Poiquilotermismo na Preguiça-Ai (Bradypus tridactylus L.). Arq. Cir. Clin. Exp., 5, 1941. São Paulo.

34. Algumas experiências fisiológicas com o Cafesterol e seus derivados. Bol. Fac. Fil. Ci. Letr. USP Química, 1:2-9, 1941. São Paulo (Em col. H. Hauptmann e Lucy Lacerda).

35. Contribuição para o estudo do sistema circulatório no Anfíbio, Siphonops annulatus. Bol. Fac. Fil. Ci. Letr. USP., Zool., 5:209-233, 1941. S. Paulo.

36. Sobre o aparelho de apreensão dos alimentos e sobre a glândula do intestino médio de Ostracodo. Ação das substâncias colinergéticas. Bol. Fac. Fil. Ci. Letr. USP., Zool., 6:107-152, 1942. São Paulo. 
37. The tail of a fish larva as respiractory organ. Nature n. 3771, 1942. Londres.

38. Comentários sobre Quadrupes e Serpentes. Cap. 1-15 do Livro IV da "Historia Naturalis Brasiliae" de Jorge MarcGrave, trad. pp. 78-88, 1942. São Paulo.

39. Comentários sobre Peixes. Cap. 19-22 do Livro IV da "História Naturalis Brasiliae” de Jorge MarcGrave, trad. bras. pp. 51-69, 1942. São Paulo. (Em col. J. P. Carvalho).

40. Comentários sobre Crustáceos, Moluscos e Equinodermes. Cap. 19-22 do livro IV da "Historia Naturalis Brasiliae" de Jorge MarcGrave, trad. bras., pp. 61-65, 1942. São Paulo.

41. An incretory in the of Lygia exotica Roux (Crustacea-Decapoda). Proc. $8^{\text {th }}$ American Sci. Congr., 3:487-490, 1942. Washington.

42. Sobre a ocorrência de Acetilcolina no tecido cardíaco de Callinectes danae Smith e seu efeito sobre o coração deste Decapodo. Bol. Fac. Fil. Ci. Letr. USP., Zool., 7:261-304, t. 1-4, 1943 . São Paulo.

43. Fisiologia e Zoologia. Novos Rumos. Bol. Fac. Fil. Ci. Letr. USP., Zool., 8:1-16, 1944. São Paulo.

44. Solução perfusora para Callinectes danae Smith. Bol. Fac. Fil. Ci. Letr. USP., Zool., 8:151-162, t. 1, 1944. São Paulo.

45. Solução perfusora para Callinectes danae Smith. Alguns efeitos de seus constituintes iônicos sobre o coração. Bol. Fac. Fil. Ci. Letr. USP., Zool., 9:5-24, 1945. São Paulo.

46. Sobre a proteção da água contra o oxigênio do ar com auxílio de óleos minerais e vegetais. Bol. Fac. Fil. Ci. Letr. USP., Zool., 10:135, 1945. São Paulo.

47 Sobre o consumo de oxigênio de alguns peixes fluviais neotrópicos (Cichlidae: Cichla e Astronotus). Bol. Fac. Fil. Ci. Letr. USP., Zool., 11:333-336, 1946. São Paulo.

48. Sobre a biologia de alguns peixes de respiração aérea (Lepidosiren paradoxa e Arapima gigas). Bol. Fac. Fil. Ci. Letr. USP., Zool., 11:225-286, 1946. São Paulo.

49. Sobre a construção dos ninhos e a reprodução de alguns peixes neotrópicos (Cichlidae - gen. Cichla e Astronotus). Bol. Fac. Fil. Ci. Letr. USP., Zool., 11:357-382, 1946. São Paulo.

50. Sobre o consumo de oxigênio pelos animais aquáticos. São Paulo. I.a Reunião da Sociedade de Biologia do Brasil, 1946. São Paulo.

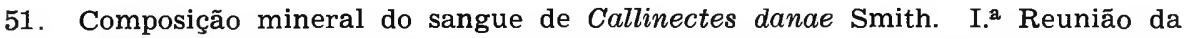
Sociedade de Biologia do Brasil, 1946. (Em col. R. S. Pereira).

52. Nota sobre a geologia de alguns crustáceos decápodos marinhos de São Paulo. Bol. Fac. Fil. Ci. Letr. USP., Zool., 11:383-393, 1946. São Paulo. (Em col. R. S. Pereira).

53. Sobre biologia de Gonileptes Horrduz Kirby (Arachneda-Opiliones). I. ${ }^{2}$ Reunião da Soc. Biol. Brasil., 1946. São Paulo. (Em col. Benedito Soares). 
54. Metabolism of the Lungfish. Lepidosiren paradoxa and the Limibless. Amphibian Typhlonectes compressicauda. $7^{\text {th }}$ Int. Physiol. Congr., pp. 381-382, 1947. Inglaterra.

55. Metabolismo respiratório dos peixes de respiração aérea (Lepidosiren paradoxa Fitz). Bol. Fac. Fil. Ci. Letr. USP., Zool., 12:43-50, 1947. S. Paulo.

56. Metabolismo respiratório do Anfíbio-Gymnophiona - Typhlonectes compressicauda compressicauda (Dum. e Bibr.). Bol. Fac. Fil. Ci. Letr. USP., Zool., 12:51-56, 1947. São Paulo.

57 Metabolismo respiratório do Anfíbio-Gymnophiona Typhlonectes compressicauda (Dum. e Bibr.). Bol. Fac. Fil. Ci. Letr. USP., Zool., 12:51-56, 1947. São Paulo.

58. Influência do anestésico sobre a coagulação do sangue de Crustáceos. Ir. ${ }^{a}$ Reunião da Soc. Biol. Brasil., 1948. Belo Horizonte.

59. O efeito do número sobre o consumo de oxigênio por animais aquáticos. II. ${ }^{a}$ Reunião Soc. Biol. Brasil., 1948. Belo Horizonte. (Em col. D. Valente).

60. Respiração e reprodução de peixes neotrópicos. II ${ }^{a}$ Reunião Soc. Biol. Brasil., 1948. Belo Horizonte. (Em col. A. Maranhão).

61. Influência da temperatura sobre o consumo de oxigênio pelas planárias. Bol. Fac. Fil. Ci. Letr. USP., Zool., 13:329-334, 1948. São Paulo. (Em col. M. D. Ungaretti).

62. Os morcegos e o radar. Cha. Quint., 78(5):285-288, 1948. S. Paulo.

63. O ensino das ciências naturais nas escolas secundárias. Cultus n. 1, pp. 1920, 1949. S. Paulo.

64. Concurso para a cadeira de História Natural no Magistério Secundário. Ci. Cult., 1(1-2):40-42, 1949. São Paulo.

65. O ensino da História Natural nos cursos secundários. Cí. Cult., 1(4) :213-214, 1949. São Paulo.

66. Microrespirômetro de Schollander e Ed'wards (demonstração). III.a Reunião da Soc. Biol. Brasil., 1949. Salvador.

67. Reação de Invertebrados às drogas coli e adrenérgicas. III. ${ }^{a}$ Reunião da Soc. Biol. Brasil., 1949. Salvador.

68. Reação do coração dos Escorpiões do gênero Tityus a desenervação e às drogas coli e adrenérgicas. Bol. Fac. Fil. Ci. Letr. USP., Zool., 14:325-334, 1948. São Paulo. (Em col. Benedito A. M. Soares).

69. Digestão nos Invertebrados. Com. I.a R.A.S.B.P.C., Ci. Cult., 2(1):35, 1950. São Paulo.

70. Anaerobiose nos Vermes. Com I. ${ }^{a}$ R.A.S.B.P.C., Ci. Cult., 2(1):37, 1950. São Paulo. (Em col. M. D. Ungaretti).

71. On the Branchiostoma (Amphioxus) of the coast of São Paulo. Bol. Fac. Fil. Ci. Letr. USP., Zool., 16:235-238, 1950. São Paulo. 
72. Achegas ao dicionário dos animais do Brasil de Rodolfo von Ihering. Bol. Instituto Paulista de Ocean., 1(2):103-116, 1950. São Paulo. (Em col. J. P. Carvalho).

73. Respiração dos Vertebrados. Cultus, (2):1-3, 1950. São Paulo.

74. Reencontro de Balanoglossus gigas Fr. Müller no litoral brasileiro. Bol. Inst. Ocean., 1(1):135-138, 1950. São Paulo.

75. Sobre a composição de alguns elementos de soro sanguíneo da Treme-Treme (Narcine brasiliensis Olfers). Com. II. ${ }^{a}$ R.A.S.B.P.C., Ci. Cult., 2(4):288289. (Em col. Antonia Bruno).

76. Sobre a fisiologia da broca do café (Hypothemus lamprei, Ferrari, 1867). Com. III. ${ }^{a}$ R.A.S.B.P.C., Ci. Cult., 2(4):289-290, 1950 . S. Paulo.

77 Cholinesterase Activity of electriccorgan of Narcine braziliensis (Olfers). Bol. Fac. Fil. Ci. Letr. USP., Zool., 16:321-327, 1951. São Paulo. (Em col. E. G. Mendes).

78. Balanoglossus gigas Fr. Müller rediscovered on the Brazilian Coast. Nature, 167 : $730,1951$. Londres.

79. Sensibilidade do músculo longitudinal radial de Holothuria à acetilcolina (efeito da acetilcolina). Ci. Cult., 3(1):41-42, 1951. S. Paulo.

80. O primeiro centenário do nascimento de Herman von Ihering (1850-1950). Ci. Cult., 3(1):52-61, 1951. São Paulo.

81. Cuénot. Ci. Cult., 3(2):136-137, 1951. São Paulo.

82. Sobre as fibras nervosas gigantes de Balanoglossus gigas Enteropneusta. Com. III. ${ }^{a}$ R.A.S.B.P.C., Ci. Cult., 3(4):267, 1951. São Paulo. (Em col. L. Forneris).

83. Sobre a estrutura do "fígado" e as funções "hepáticas" de Balanoglossus gigas. Com III.a R.A.S.B.P.C., Ci. Cult., 3(4):270, 1951. S. Paulo. (Em col. T. Bjornberg).

84. Animais venenosos. Com. III. R.A.S.B.P.C., Ci. CuIt., 3(4):275, 1951. S. Paulo.

85. Alguns dados sobre a estrutura de Australorbis glabratus. Com III.a R.A. S.B.P.C., Ci. Cult., 3(4), 1951. São Paulo. (Em col. A. A. A. Lopes e J. Bobin Jr.).

86. Sobre a musculatura de Balanoglossus gigas Enteropneusta, e sua reação às drogas coli e adrenérgicas. Com. III. ${ }^{a}$ R.A.S.B.P.C., Ci. Cult., 3(4): 286, 1951. São Paulo.

87. Atividades do Departamento de Fisiologia Geral e Animal em 1951. An. Fac. Fil. Ci. Letr. USP., 12 pp., 1951. São Paulo.

88. Sobre a ação da acetylcholina sobre o esôfago da Aplysia. Com. III. ${ }^{a}$ R.A. S.B.P.C., Ci. Cult., 3(4):287, 1951. São Paulo. (Em col. N. Chaves).

89. Metabolismo respiratório de Australorbis glabratus. Com. IIr.a R.A.S.B. P.C., Ci. Cult., 3(4):312, 1951. S. Paulo. (Em col. C. A. Edwards). 
90. Sobre a taxonomia dos Enteropeneustas brasileiras. Com. IV.a R.A.S.B. P.C., Ci. Cult., 4(3-4):104, 1952. São Paulo. (Em col. L. Forneris).

91. Atividade muscular de Holothuria grisea. Com. IV.a R.A.S.B.P C., Ci. Cult., 4(3-4):110, 1952. São Paulo. (Em col. C. A. Pantin).

92. Reação do músculo rađial de Holothuria às drogas colinérgicas. Com. IV.a R.A.S.B.P.C., Ci. Cult., 4(3-4):129, 1952. Sãc Paulo.

93. Achegas ao Dicionário dos Animais do Brasil de Rodolfo von Ihering. Letra B, Bol. Inst. Oceanogr., 3(1-2):189-199, 1952. São Paulo. (Em col. J. P. Carvalho).

94. Psicologia Animal. Separata do livro: "Psicologia Moderna", pp. 101-131, 1. ${ }^{\circledR}$ ed., Livr. Agir, 1953. São Paulo.

95. Use of Holothuria grisea for Acetilcholina assays if electric organ extracts from Narcine brasiliensis (Olfers). Physiologia Comparata e Oecologia, 3(1): 52-56, 1953. Dan Haag. (Em col. A. Ambache).

96. Estudo comparativo da ultraestrutura do músculo estriado com o microscópio eletrônico. Ci. Cult., 5(1):27-31, 1953. São Paulo. (Em col. G. A. Edwards, P. S. Santos e H. L. S. Santos).

97 Enteropneustas brasileiros. Bol. Fac. Fil. Ci. Letr. USP., Zool., 18:5-50, 1953. São Paulo. (Em col. L. Forneris).

98. Muscular action in Holothuria grisea. Bol. Fac. Fil. Ci. Letr. USP., Zool., 18:51-60, 1953. (Em col. C. A. F. Pantin).

99. O ensino das ciências biológicas nas escolas. Ci. Cult., 5(2):98, 1953 . São Paulo.

100. Muscular action in Holothuria grisea. Bol. Fac. Fil. Ci. Letr. USP., Zool., 18:51-60, 1953. São Paulo. (Em col. C. A. F. Pantin).

101. Reação do coração de Vertebrados Pecilotermos à adrenalina e à novadronalina. Com. V.a R.A.S.B.P.C., Ci. Cult., 5(4):204, 1953. São Paulo.

102. Fisiologia Comparada dos Mecanismos neuro-humorais (Insetos e Equinodermes). Com. V.a R.A.S.B.P.C., Ci. Cult., 5(4):206, 1953. São Paulo. (Em col. E. G. Mendes).

103. Ultraestrutura do músculo estriado de Invertebrados. Com. Va R.A.S.B. P.C., Ci. Cult., 5(4):207, 1953. São Paulo. (Em col. G. A. Edwards, P. S. Santos e N. S. Santos).

104. Estudo do músculo estriado de Reptilia com o microscópio eletrônico. V.a R.A.S.B.P.C., Ci. Cult., 5(4):208, 1953. São Paulo. (Em col. P. S. Santos, A. A. Hoge e G. A. Ed'wards).

105. Microscópio eletrônico de fibras colágenas. Com V.a R.A.S.B.P.C., Ci. Cult., 5(4):209, 1953. São Paulo. (Em col. H. L. S. Santos, P. S. Santos, G. A. Edwards).

106. Studies on the Action of Acetylcholine in Insecta and Echinoderme. Abstr. Com. XIX Intern. Physiol. Congr. Montreal, Canadá, 1953.

107. Sobre a contração do músculo longitudinal de Holothuria grisea. Com. VI.a R.A.S.B.P.C., Ci. Cult., 6:193, 1954. São Paulo. 
108. Observaçōes sobre a digestão nas Planárias. Com. VI.a R.A.S.B.P.C., Ci. Cult., 6(4):193, 1954. São Paulo.

109. A estomocorda dos Enteropneustas (Balanoglossus). Com. VI.a R.A.S.B. P. C., Ci. Cult., 6(4):201, 1954. São Paulo.

110. Electron microscope studies of Insect Muscles. I. Flight and Coxal Muscle of Hydrophilus picens. Ann. Entomol. Soc. Amer., 47(2):343-354, 1964. (Em col. G. A. Edwards, P. S. Santos e H. L. S. Santos).

111. Electron microscope studies of Insect Muscle. II. Flight and leg muscle of Belostoma and Periplaneta. Ann. Entomol. Soc. Amer., 47(3):459-467. 1954. (Em col. G. A. Edwards, P. S. Santos, H. L. S. Santos).

112. Electron microscope studies of Insect Muscle. III. Variations in ultraestructure. Bol. Fac. Fil. Ci. Letr. USP., Zool., 19:391-404, 1 tab., 14 fig., 1954. São Paulo. (Em col. G. A. Edwards, P. S. Santos, H. L. S. Santos). 113. A importância e necessidades da sistemática na pesquisa biológica. Ci.
Cult., 6(1):25-26, 1954. São Paulo. 114. Um século de publicações científicas. Ci. Cult., 6(2):91-92, 1954. São
Paulo.

115. Laboratórios de Biologia Marinha. Ci. Cult., 6(3):107-109, 1954. S. Paulo.

116. Estudos eletro-microscópicos de músculos estriados de Répteis. Mem. Inst. Butantan, (26) : $169-175,1954$. S. Paulo. (Em col. G. A. Edwards, P. S.
Santos, A. R. Hoge e Vallejo-Freire) 117. Electron microscopy of Insect Tracheal Structure. An. Acad. Bras. Ci.,
$2(26): 309-315,1954$. Rio de Janeiro.

118. Apontamentos de Zoologia. Vertebrados, $1 .^{\text {a }}$ ed., 128 pp., 1954. S. Paulo (2. ${ }^{a}$ ed. ilustrada, 111 pp., S. Paulo, 1956).

119. Apontamentos de Zoologia. Invertebrados (Artropodes-Anelídeos), 157 pp..
1954. São Paulo. 120. Apontamentos de Zoologia. Invertebrados (Acantocéfalos, Platelmintos, Ne-
mertinos). 64 pp., 1954. S. Paulo.

121. Sinópse das atividades do Departamento de Fisiologia Geral e Animal, 1954-
1955. S. Paulo.

122. Uma contribuição ao conhecimento da estrutura ultrafina e das propriedades químicas de fibrilas do tecido conjuntivo animal. Mem. Inst. Butantan, 27:1-39, 1955. S. Paulo. (Em col. P. S. Santos, G. A. Edwards, A. R.
Hoge).

123. Dirigismo. Ci. Cult., 7(1):1-2, 1955. S. Paulo.

124. Renúncia à Pesquisa. Ci. Cult., 7(2):65-66. 1955. São Paulo.

125. A SBPC e as Universidades. Ci. Cult., 7(3):125-126, 1955. São Paulo.

126. Balanoglossus do Estado de S. Paulo. Com. VII. ${ }^{a}$ R.A.S.B.P.C., Ci. Cult., I(3):158, 1955. São Paulo. (Em col. M. A. Esquibel). 
127 Sobre a ação dos íns na mecânica uterina. Com. VII. ${ }^{a}$ R.A.S.B.PC., Ci. Cult., 7(3):160, 1955. São Paulo. (Em col. C. N. Grinkraut).

128. Holoturias do Recife. VII.a R.A.S.B.P.C., Ci. Cult., 7(3):166, 1955. São Paulo. (Em col. A. A. A. Lopes).

129. Professor W. C. A. Alee. Ci. Cult., 7(3):182, 1955. São Paulo.

130. Atividades da S.B.P.C. e seus sócios. Ci. Cult., 7(4):193-194, 1955. São Paulo.

131. A carreira de pesquisador. Ci. Cult., 8(1):1-2, 1955, S. Paulo.

132. Perspectivas em Biologia Marinha. Ci. Cult., 8(2):114-116, 1956 . S. Paulo.

133. Ensino de Biologia nas Escolas Secundárias. Ci. Cult., 8(2):119-120, 1956. S. Paulo.

134. Laboratórios de Biologia Marinha e Oceanografia na Costa Pacífica Americana. Ci. Cult., 8(2):121, 1956. São Paulo.

135. Regeneração de Planárias. Com. VIII.̊ R.A.S.B.P.C., Ci. Cult., 8(3):165, 1956. São Paulo.

136. Sobre a contração da cloaca de Holothuria grisea. Com. VIII.a R.A.S.B. P.C., Ci. Cult., 8(3):169, 1956. São Paulo. (Em col. A. A. A. Lopez).

137. Neurosecreção em Isopodes. Com. VIII.a R.A.S.B.P.C., Ci. Cult., 8(3): 169, 1956. São Paulo. (Em col. R. M. Correia).

138. Fisiologia do coração dos Répteis. Com. VIII.a R.A.S.B.P.C., Ci. Cult., 8(3):170, 1956. São Paulo.

139. Exames vestibulares. Ci. Cult., 8(3):194, 1956. São Paulo.

140. Laboratórios de Biologia Marinha de Miami. Ci. Cult., 8(3):199, 1956. São Paulo.

141. Carreira Universitária. Ci. Cult., 8(4):211, 1956. S. Paulo.

142. Marine Biological Studies in Brasil. Publ. Dept. Fisiol. Geral e Animal da USP., 4 pp., 1956. S. Paulo.

143. Faculdades isoladas no Interior. Ci. Cult., 9(1):1, 1957. S. Paulo.

144. Estudos sobre a ultraestrutura de músculos de Invertebrados. Ci. Cult., 9(1):21-26, 1957. S. Paulo. (Em col. B. Oliveira F.o e P. S. Santos).

145. Neurosecreção - Aspectos Gerais. Simpósio. Com. IX.a R.A.S.B.P.C., Ci. Cult., 9(3):140, 1957. São Paulo.

146. Neurosecreção em Anfíbios. Com. IX. ${ }^{a}$ R.A.S.B.P.C., Ci. Cult., 9(3):140, 1957. S. Paulo. (Em col. C. Silva Jr.).

147. Distribuição das glândulas secretoras de suco gástrico no estômago de Anfíbios. Com. IX.a R.A.S.B.P.C., Ci. Cult., 9(3):142-143. S. Paulo. (Em col. L. Yamashita).

148. Contribuição para o estudo da fibra muscular cardiaca dos répteis. Com. IX. ${ }^{a}$ R.A.S.B.P.C., Ci. Cult., 9(3):148, 1957. S. Paulo. 
149. Sobre o metabolismo respiratório dos Onicóforos. Com. IX. ${ }^{a}$ R.A.S.B.P.C., Ci. Cult., 9(3):150, 1957. S. Paulo. (Em col. E. G. Mendes).

150. Ação da acetilcolina e do curare sobre a fibra muscular de Invertebrados. Com. IX.a R.A.S.B.P.C., Ci. Cult., 9(3):166, 1957. S. Paulo. (Em col. M. Silveira).

151. Anatomia funcional do coração de Répteis. Fol. Clin. Biol., 27(1):201, 1957.

152. Contribution à l'étude de la composition chemique du sang de certains sélaciens du Brésil. Bol. Fac. Fil. Ci. Letr. USP., Zool., 21:85-92, 1957. S. Paulo.

153. On the action of ions of the uterine mechanics. Bol. Fac. Fil. Ci. Letr. USP., Zool., 21:123-124, 1957. São Paulo. (Em col. C. H. Grinkraut).

154. Cientistas de segunda classe. 'Ci. Cult., 9(4), 1957. S. Paulo.

155. Estrutura das Universidades. Ci. Cult., 10(1):1-3, 1958. S. Paulo.

156. Dez anos pelo Progresso da Ciência. Ci. Cult., 10(2):53-54, 1958. S. Paulo.

157. The oxygen consumption of "Onychophora" and its relation to size temperature and oxygen tension. Rev. Bras. Biol., 18(2):129-142. Rio de Janeiro. (Em col. E. G. Mendes).

158. The teaching of Zoology. XVth Intern. Congr. Zool. Sect. I, Paper 12, 1958. Londres.

159. Perspectivas em Biologia Marinha. Com. X.a R.A.S.B.P.C., Ci. Cult., 10(3) : 156, 1958. S. Paulo.

160. Ultraestrutura do tecido muscular de um crustáceo decápode. Com. X. ${ }^{a}$ R.A.S.B.P.C., Ci. Cult., 10(3):153, 1958. S. Paulo. (Em col. R. Lavallard, P. S. Santos e H. S. Santos).

161. Observações preliminares sobre a ultraestrutura da musculatura longitudinal de larvas de borboleta. Com. X.a R.A.S.B.P.C., Ci. Cult., 10(3):153, 1958. S. Paulo. (Em col. B. Oliveira F. ${ }^{\circ}$ ).

162. Sobre a fisiologia do músculo longitudinal de Holothuria grisea. Bol. Fac. Fil. Ci. Letr. USP., Zool., 22:75-97, 1958. S. Paulo. (Em col. A. A. A. Lopez).

163. Sobre aspectos interessantes da anatomia do coração de Jibóia (Constictor constrictor). Com. XI. ${ }^{a}$ R.A.S.B.P.C., Ci. Cult., 11(3):143. S. Paulo.

164. A organização sub-microscópica de músculo longitudinal de Holotúria. Com. XI.a R.A.S.B.P.C., Ci. Cult., 11(3):152, 1959. S. Paulo.

165. O emprego do azul de metileno no estudo do sistema nervoso intercardíaco de Invertebrados. Com. XI.a R.A.S.B.P.C., Ci. Cult., 11(3):155, 1959. S. Paulo.

166. Arquitetura da cadeia nervosa de larva do lepidoptero (Ecpantheria indecisa). Com. XI. a R.A.S.B.P.C., Ci. Cult., 11(3):155-156, 1959. S. Paulo. (Em col. O. A. Pereira). 
167. Sobre a respiração intestinal do Cascudo. Com. VII.a R.A.S.B.P C., Ci. Cult., 12(2):85, 1960. S. Paulo. (Em col. L. Petrini).

168. Sobre Balanoglossus gigas. Com. XII.a R.A.S.B.P.C., Ci. Cult., 12(2): 86, 1960. S. Paulo. (Em col. I. Riepenhoff).

169. Sobre um órgão novo do Peixe Cascudo (Plecostomus sp.) e sua provável função. Com. XII. a R.A.S.B.P.C., Ci. Cult., 12(2):87, S. Paulo, 1960.

170. Sobre a presença de quinina dos animais. Com. XII.a R.A.S.B.P.C., Ci. Cult., 12(2):87, 1960. S. Paulo. (Em col. G. S. Alterthum, W. Rossi).

171. Tingui de Irapuã, tóxico para peixes. Com. XII.a R.A.S.B.P.C., Ci. Cult., 12(2):93, 1960. S. Paulo. (Em col. L. M. Aguiar).

172. Sobre a presença da cloaca na respiração intestinal no Cascudo (Loricadidae: Plecostomus elecostomus (Linn.). Bol. Fac. Fil. Ci. Letr. USP., Zool., 23:5-24, 1960. S. Paulo. (Em col. L. M. Petrini).

173. Vantagens do tempo integral. Relatório apresentado na Reunião de Representantes de Instituições Latinoamericanas. Caracas, 1960.

174. George Alfred Edwards. In memoriam. Ci. Cult., 13(1):35-40, $1961 . \mathrm{S}$. Paulo.

175. Ainda sobre um órgão novo do Peixe Cascudo (Plecostomus sp.) e sua provável função. Com. XIII.a R.A.S.B.P.C., Ci. Cult., 13, 1961. S. Paulo.

176. Sobre a ocorrência de Strophocheilidas (Molusco Gastropode) do Rio Grande do Sul. Com. XIII. ${ }^{a}$ R.A.S.B.P.C., Ci. Cult., 13, 1961. S. Paulo. (Em col. J. A. Petersen).

177. Observação sobre a regeneração da Planaria dugesia trigrina. Com. XIII. ${ }^{2}$ R.A.S.B.P.C., Ci. Cult., 13, 1961. S. Paulo. (Em col. M. Silveira).

178. Sobre o funcionamento rítmico da glândula do intestino médio e do intestino propriamente dito de Strophocheilus (Gastropode-Pulmonado). Com. XIII.a R.A.S.B.P.C., Ci. Cult., 13, 1961. S. Paulo. (Em col. C. Volkmer).

179. Medida da atividade da musculatura somática e visceral de Invertebrados. Com. XIII.a R.A.S.P.B.C., Ci. Cult., 13, 1961. S. Paulo.

180. Sobre a controvertida presença de túbulos de Malpighi em escorpiões. Com. XIV. ${ }^{a}$ R.A.S.B.P.C., Ci. Cult., 14, 1962. S. Paulo. (Em col. J. E. Lunetta).

181. Osmoralidade do músculo longitudinal de Holothuria grisea. Com. XIV.a R.A.S.B.P.C., Ci. Cult., 14, 1962. S. Paulo. (Em col. G. Sestini).

182. Etologia de Balanoglossus gigas Fr. Müller. Com. XIV.a R.A.S.B.P.C., Ci. Cult., 14, 1962. S. Paulo. (Em col. B. Jones, J. A. Petersen).

183. Sobre a fauna coexistente com Balanoglossus gigas Fr. Müller. Com. XIV.a R.A.S.B.P.C., Ci. Cult., 14, 1962. S. Paulo. (Em col. J. A. Petersen).

184. On a bioosay for acetylcholine and on some properties of the longitudinal muscles of Holothuria grisea (Echinodermata). Bol. Fac. Fil. Ci. Letr. USP., Zool., 24:5-10, 1962. S. Paulo. 
185. Sobre a ocorrência de Strophocheilidae (Molusco-Gastrópode) no Rio Grande do Sul. Bol. Fac. Fil. Ci. Letr. USP., Zool., 24:31-42, 1962. S. Paulo. (Em col. J. A. Petersen).

186. Fisiologia da circulação. Experiências com mexilhões e serpentes. Com. XV. ${ }^{a}$ R.A.S.B.P.C., 1963. S. Paulo.

187. Redescrição de B. gigas e notas sobre a ecologia deste Enteropneusto. Com. XVa R.A.S.B.P.C., 1963. S. Paulo. (Em col. M. S. Campos).

188. Sobre Ascidias novas para o litoral brasileiro. Com XVa R.A.S.B.P.C., 1963. S. Paulo. (Em col. S. R. Almeida).

189. Primeiros resultados sobre o ciclo sexual do Mexilhão (Mytilus perna) comum ao litoral de S. Paulo. Com. XV.a R.A.S.B.P.C., 1963. S. Paulo. (Em col. J. E. Lunetta).

190. Sobre a musculatura de Balanoglossus gigas. Com. XV.a R.A.S.B.P.C., 1963. S. Paulo. (Em col. S. Schuster e W. F. Ramos).

191. Reencontro de B. gigas no mesmo local de sua primeira captura por Fr. Müller em 1883. Com. XV.a R.A.S.B.P.C., 1963. S. Paulo. (Em col. J. A. Petersen).

192. Enteropneustos. Hist. Nat. Org. Aquat. do Brasil, pp. 309-316, 1964.

193. Fisiologia dos Animais Marinhos. Hist. Nat. Org. Aquat. do Brasil, pp. 1930, 1964. S. Paulo. (Em col. A. A. A. Lopez).

194. Acrania (Protocordados). Hist. Nat. Org. Aquat. do Brasil, pp. 305-308, 1964. S. Paulo.

195. O ensino da Fisiologia. Ci. Cult., 16(4):379, 1964. Sāo Paulo.

196. Simpósio de Biologia Marinha. Ci. Cult., 17(1):43-44, 1965. S. Paulo.

197. Biochemical studies on the snail Strophocheilus oblongusmusculus (Becquaert). Com. Biochem. Physiol., 14:35-42, 1965.

198. Iodine: Accumulation by Balanoglossus gigas. Science, 150(3700):1182-1183, 1965. S. Paulo.

199. Physiological aspects of the ecology of the mussel Mytilus perna L. An. Acad. Bras. Ci., Supl., 37:176-177, 1965. Rio de Janeiro.

200. Biochemical studies on the giant earthworm Glossocolex gigantes (Leuckart). Comp. Biochem. Phys., 16:491-496, 1965. S. Paulo.

201. Alguns aspectos da ecologia de Echinodermata. An. Acad. Bras. Ci., 37. supl:167-170, 1965. S. Paulo. (Em col. J. A. Petersen, Liu Pin Yi).

202. The actions of Acetylcholine on the isolated of Mytilus perna. Bol. Fac. Fil. Ci. Letr. USP., Zool., 25:5-12, 1965, 2 t. S. Paulo. (Em col. J. Khouri).

203. Biochemical studies in a Eunicid (Polychaete) of the littoral of São Paulo, Brazil. Comp. Biochem. Physiol., 17:535-551, 1966.

204. Toxic Marine Invertebrates - Venomous and noxious fishes of fresh water. Mem. Inst. Butantan, Simp. Intern., 33(1):31-34, 1966. 
205. Aspectos interessantes do encéfalo de grandes peixes da família siluridae (Jaú, Pintado e Mandi) dos rios do Estado de São Paulo. Com. XIX. ${ }^{a}$ R.A. S.B.P.C., Ci. Cult., 19(2):350, 1967.

206. Algumas observações acerca da histologia e da histoquímica do sistema neurosecretor caudal de peixes Teleósteos. Com. XIX. R.A.S.B.P.C., Ci. Cult., 19(2):357, 1967. (Em col. O. F. Martins).

207 Algumas observaçōes histológicas sobre o órgão último branquial de Peixes Teleósteos. Com. XIX.a R.A.S.B.P.C., Ci. Cult., 19(2):361, 1967. (Em col. M. Di Lascio Teraoka).

208. Neurosecreção em anelídeos poliquetos. Com. XIX.a R.A.S.B.P.C., Ci. Cult., 19(2):366, 1967. (Em col. R. H. Canteras).

209. Uma nova função da uróhipofise de peixes Teleósteos. Com. XIX.a R.A. S.B.P.C., Ci. Cult., 19, 1967. (Em col. O. F. Martins).

210. Neurosecreção em anelídeos oligoquetos. Com XIX.a R.A.S.B.P.C., Ci. Cult., 19(2):385, 1967. (Em col. M. T. C. Faria).

211. Ação do cálcio e efeito das drogas sobre o músculo liso despolarizado de Moluscos Opistrobrânquios. Com. XIX.a R.A.S.B.P.C., Ci. Cult., 19(2): 406, 1967. (Em col. I. N. Cipolli).

212. Considerações sobre a acumulação de iôdo por invertebrados e protocordados marinhos. Com. XIX.a R.A.S.B.P.C., Ci. Cult., 19(2):412, 1967.

213. Associação de Copépodes e Moluscos Opistobrânquios. Com. XIX.a R.A. S.B.P.C., Ci. Cult., 19(2):432, 1967. (Em col. Ivete N. Cipolli).

214. Sobre o programa biológico internacional. Reunião promovida pela Academia Nacional de Ciências dos Estados Unidos. Com. XX.a Reunião Anual da S.B.P.C., Ci. Cult., 20(2):339, 1968.

215. O encéfalo de peixes da família Pimelodidae (Phractocephalus sp.). Com.

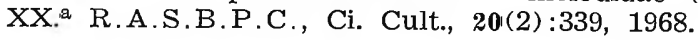

216. Sobre a digestão extra-intestinal de ouriço do mar (Lytechinus variegatus).

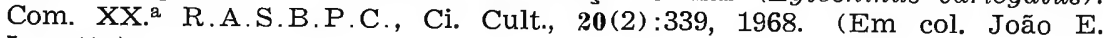
Lunetta).

217 Comportamento de peixes siluriformes do gênero Rhamdia e baixas temperaturas. Com. R.A.S.B.P.C., Ci. Cult., 20(2):380, 1968. (Em col. Olga F. Martins).

218. Comportamento e metabolismo respiratório da Lepidosiren paradoxa do Amazonas. Com. XX.a R.A.S.B.P.S., Ci. Cult., 20(2):393, 1968. Em col. M. D. Pérez-Gonzalez).

219. Reação da fibra muscular lisa de moluscos opistobrânquios à acetilcolina após despolarização da membrana. Com. XX. ${ }^{\circledR}$ R.A.S.B.P.C., Ci. Cult., 20(2) : 440, 1968.

220. Calcium and the effects of drugs on smooth muscle of the Oesophagus of Aplysia brasiliana (Mollusc-Opistobranch). Bol. Fac. Fil. Ci. Letr. USP., Zool. Biol. Mar., N.S., 26:5-18, 1969.

221. O bloqueio da acetilcolina pela atropina no músculo longitudinal de Holo- 
thuria grisea. XXI. ${ }^{2}$ R.A.S.B.P.C., Ci. Cult. (Em col. Ana Amelia Ancona Lopez).

222. Efeitos dos ions $\mathrm{Ca}^{2}+$ sobre a contração das fibras musculares lisas de invertebrados marinhos. XXI.a R.A.S.B.P.C., Ci. Cul., 21(2):504, 1969.

223. Sensibilidade dos músculos dorsais da sanguessuga terrestre, Liostoma (Wagler, 1831) à acetilcolina. XXI.a R.A.S.B.P.C., Ci. Cult., 21(2):540, 1969. (Em col. Paulo Pasquarelli).

224. Aspectos ecológicos e físiológicos da fauna entre-marés da praia Lodoso Continental e da Arenosa Insular. XXII.a R.A.S.B.P.C., 22:286-287, 1970.

225. Respostas do músculo dorsal de sanguessuga terrestre Liostoma sp. a drogas de uso farmacológico. XXII. ${ }^{a}$ R.A.S.B.P.C., Ci. Cul., 22:358. (Em col. P. Pasquarelli).

226. Interferência do sistema neurosecretor caudal sobre o metabolismo de hidratos de carbono. XXII. ${ }^{a}$ R.A.S.B.P.C., 22:359, 1970. Em col. Olga M. Mimura).

227 Resposta do coração isolado de Aplysia brasiliana (molusco-opistobrânquio) a várias drogas de uso farmacológico. XXII. ${ }^{a}$ R.A.S.B.P.C., Ci. Cult., $22: 359,1970$.

228. Influência da salinidade sobre a contração da musculatura lisa do esôfago de Aplysia brasiliana (Molusco Gastropode-Opistobrânquio, tectibrânquio). XXII. ${ }^{\text {a }}$ R.A.S.B.P.C., Ci. Cult., 22:360, 1970. (Em col. W. M. Leahy).

229. Sobre o teor glicêmico da piramboia (Lepidosiren paradoxa). XXII. ${ }^{a}$ R.A. S.B.P.C., Ci. Cult., 22:361, 1970.

230. Influência de ions na bioluminescência de Renilla sp. XXII. ${ }^{a}$ R.A.S.B.P.C., Ci. Cult., 22:380, 1970. (Em col. Ana Maria Vilela Soares).

231. Alguns aspectos da regulação osmótica em asteróides na região tropical. XXII.a R.A.S.B.P.C., Ci. Cult., 22:380, 1970. (Em col. M. Comenale, M. V. V. Sella).

232. Sobre a velocidade locomoção de asteróides da região tropical. XXII. ${ }^{a}$ R.A. S.B.P.C., Ci. Cult., 22:381, 1970. (Em col. Amalia S. Kalckmann e D. Lamoglia).

233. Efeito da acetilcolina e da atropina sobre o músculo longitudinal do corpo de Holothuria grisea (Equinoderme). XXII. ${ }^{a}$ R.A.S.B.P.C., Ci. Cult., 22: 375, 1970. (Em col. A. L. Viana, M. H. dos Reis).

234. Sobre o efeito de Cálcio++ no funcionamento do esófago de Aplysia brasiliana (Molusco-Opistobrânquia). XXII.a R.A.S.B.P.C., Ci. Cult., 22:375, 1970. (Em col. W. M. Leahy).

235. Sobre a fisioecologia de alguns animais da praia da Ilha de São Sebastião. IV Congr. Bras. Zool., C 6, 1970. (Em col. O. Genofre e Godofredo Genofre).

236. Fisiologia do tegumento de trairamboia (Lepidosiren paradoxa). XXIII.a R.A.S.B.P.C., Ci. Cult., 23:298, 1971. (Em col. L. Petrini). 
237 Ação do veneno das paratoides no coração do sapo (Bufo marinus ictericus) durante a estimulação vagal. XXIII.a Reunião Anual da S.B.P.C., Ci. Cult., 23:347, 1971. (Em col. P. Pasquarelli).

239. Bioluminescência de Renilla amethystina Verill, 1864 (Coelenterata-Anthozoa), influência do ion $\mathrm{Mg}++$. XXIII. ${ }^{a}$ R.A.S.B.P.C., Ci. Cult., 23:392, 1971. Em col. Ana Maria Vilela Soares).

240. Sobre a atividade muscular e consumo de oxigênio de Chaetopterus variopedatus L. XXIII. ${ }^{a}$ R.A.S.B.P.C., Ci. Cult., 23:393, 1971. (Em col. Mercia M. D. Silva).

241. Sobre a mudança de cor em Uca sp. (Crustáceo-Decópode). XXIII. ${ }^{\text {R R.A. }}$ S.B.P.C., Ci. Cult., 23:393-394, 1971. (Em col. Godofredo C. Genofre Netto).

242. Reações do coração de moluscos opistobrânquios-nudibrânquios (Dendodroa $s p$ ) à acetilcolina e à serotonina (5-hidroxitriptamina). XXIII.a R.A.S.B. P.C., Ci. Cult., 23:396-397, 1971. (Em col. W. Markiewicz).

243. Sobre a morfologia e fisiologia do tegumento de Lepidosiren paradoxa. V Congr. Bras. Zool., p. 36, 1972. (Em col. Lina M. de P. da Silva Coelho).

244. Pressão do fluido celômico em annelídeos. V Congr. Bras. Zool., p. 36, 1972. (Em col. Ivete N. Cipolli).

245. Neurossecreção $\mathrm{em}$ Annelida - Glossoscolex saci e Eunice sebastianae. V Congr. Bras. Zool., p. 37, 1972. (Em col. M. Therezinha C. F. Monteiro).

246. Observações sobre o comportamento em aquário de um Ceriantharia (Hexacorallia) do litoral norte paulista. V Congr. Bras. Zool., p. 37, 1972. (Em col. José Carlos de Freitas).

247. O sistema neurosecretor caudal e urófise de peixe tropicais. V Congr. Bras. Zool., p. 40, 1972. (Em col. Olga M. Mimura).

248. Comportamento de Tambaky M'Boia (Lepidosiren paradoxa, Fritz) - peixe dipnóico em condições experimentais. V Congr. Bras. Zool., p. 41, 1972. (Em col. Olga M. Mimura).

249. Sobre as cromatoforotropinas de Uca sp. (Crustáceo-Decápode). V Congr. Bras. Zool., p. 54, 1972): (Em col. G. Genofre Netto).

250. Comportamento dos crustáceos Uca rapax do mangue de São Sebastião SP. V Congr. Bras. Zool., p. 54. (Em col. Silvana Moraes Marques).

251. Observações sobre Chaetopterus variopedatus Rénier, 1804 (Polychaeta) ao litoral de S. Paulo. V Congr. Bras. Zool., p. 56. (Em col. José Carlos de Freitas).

252. Fisioecologia de animais da zona entre-marés, especialmente do manguesal do litoral de São Sebastião. V Congr. Bras. Zool., p. 58.

253. Um novo Opistobranchiata no litoral paulista (Pleurobranchus gigas sp.). V Congr. Bras. Zool., p. 58. (Em col. M. Grempel).

254. Prof. Dr. Ernst Gotthelf Marcus. Bol. Inst. Bioc. Zool. Biol. Mar., 27:5-18. São Paulo.

255. Calcium and the effects of drugs on smooth muscle of the Oesophagus of 
Aplysia brasiliana (Mollusc Opisthobranch). Bol. Inst. Bioc. Zool. Biol. Mar., 27:19-24. (Em col. W. N. Leahy).

256. Sistema neurossecretor caudal em Lepidosiren paradoxa, Fritz. XXV.a R.A. S.B.P.C., 1973. Rio de Janeiro. (Em col. Olga M. Mimura).

257. Estudo histológico e histoquímico da hipófise da carpa. XXV.a R.A.S.B. P.C., 1973. Rio de Janeiro. (Em col. M. V. do Val Sella).

258. Variações da pressão do fluido celômico em glossoscolecídeos (Oligoquetos terrestres). XXV.a R.A.S.B.P.C., 1973. Rio de Janeiro. (Em col. I. V. Cipolli).

259. Contribuição ao estudo da absorção de cromo (utilização de cromo radioativo). XXV. ${ }^{a}$ R.A.S.B.P.C., 1973. Rio de Janeiro. (Em col. M. Nazareth, J. C. Barberio e R. R. Pieroni).

260. Experiências preliminares sobre a Aplisina, substância tóxica da glândula do intestino médio de Aplysia brasiliana Rang 1828 (Mollusca, Opisthobranchia). XXV a R.A.S.B.P.C., 1973. Rio de Janeiro. (Em col. J. C. Freitas).

261. Regeneração em Balanoglossus gigas (Hemichordata). XXV a R.A.S.B.P.C., 1973. Rio de Janeiro. (Em col. L. L. L. Rosa).

262. Metabolismo de acidoboleico ${ }^{125} \mathrm{I}$. Influência da proteina isolada da soja (Glicinaemax). XXV a R.A.S.B.P.C., 1973. Rio de Janeiro. (Em col. N. Sosa, M. Zuccas e J. C. Barberio).

263. Análise cinética de um sucedâneo do plasma marcado com iôdo radioativo (311I). XXV.a R.A.S.B.P.C., 1973. Rio de Janeiro. (Em col. W. G. Cova, J. C. Barberio, C. H. Mesquita, A. S. Gouvea e R. R. Pieroni).

264. Análise cinética compartimental da benziodarona marcada com ${ }^{131} \mathrm{I}$. XXV.a R.A.S.B.P C., 1973. Rio de Janeiro. (Em col. S. Shimizu, C. H. Mesquita e A. S. Gouvea). 
